\title{
Nurses' knowledge on neonatal sepsis in a tertiary care hospital in Sri Lanka
}

\author{
Pawani Hewamalage ${ }^{1}$, H V D N Siriwardhane ${ }^{1}$, A P R D Aththanayake ${ }^{1}$, Sathika Amarasekara ${ }^{2}$, \\ *Medha Weerasekera ${ }^{3}$
}

Sri Lanka Journal of Child Health, 2019; 48(4): 316-320

\begin{abstract}
Introduction: Early diagnosis of neonatal sepsis and appropriate management can prevent permanent disability and death. The knowledge of the nurses regarding sepsis and their ability to detect early cues and actions taken on suspicion of sepsis are of paramount importance in this respect.
\end{abstract}

Objective: To assess knowledge regarding neonatal sepsis among nursing officers caring for neonates at Sri Jayewardenepura General Hospital (SJGH).

Method: A descriptive cross-sectional study was done recruiting nurses from the neonatal intensive care unit (NICU), post-natal wards, paediatric ward and the labour ward of SJGH. Data were collected through an interviewer administered questionnaire and analysed using SPSS.

Results: Eighty one nursing officers from the above mentioned units participated in the study. Of the nurses, $72.8 \%$ had an overall good knowledge on neonatal sepsis and the rest $(27.2 \%)$ had a satisfactory knowledge. Ninety five percent of nurses had good or satisfactory knowledge about what neonatal sepsis meant. All participants knew bacteria as a causative organism of neonatal sepsis. Regarding the knowledge on risk factors, majority $(80.2 \%)$ had a satisfactory or greater knowledge, while the knowledge was unsatisfactory in $19.8 \%$. Of the nurses, $85.5 \%$ knew what actions to take upon identification of neonatal sepsis. All the nurses with a working experience less than 1 year, $87.5 \%$ of BSc degree holding nurses and $71.2 \%$ of nurses with NTS training had a good knowledge.

\footnotetext{
${ }^{1}$ Registrar in Paediatrics, ${ }^{2}$ Senior Registrar in Paediatrics, ${ }^{3}$ Consultant Neonatologist, Neonatal Intensive Care Unit, Sri Jayewardenepura General Hospital, Nugegoda

*Correspondence: medhaweerasekera@gmail.com

orcid.org/ 0000-0001-5065-1889
}

(Received on 21 January 2019: Accepted after revision on 22 March 2019)

The authors declare that there are no conflicts of interest

Personal funding was used for the project.

Open Access Article published under the Creative

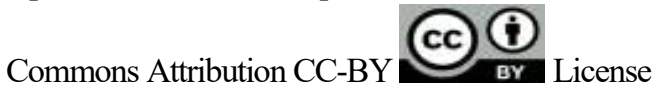

Conclusions: Seventy three percent of the nurses had an overall good knowledge on neonatal sepsis and the rest had a satisfactory knowledge. Knowledge deficiencies were identified regarding risk factors of neonatal sepsis, initial action upon identification of sepsis and the cues in identifying sepsis. No significant association was found between the knowledge on neonatal sepsis and the institute of training or duration of exposure. However, some degree of association was tracked between the unit of work and knowledge on sepsis.

DOI: http://dx.doi.org/10.4038/sljch.v48i4.8825

(Key words: Knowledge, nurses, neonatal sepsis)

\section{Introduction}

Newborn sepsis is characterized by signs and symptoms of infection such as temperature fluctuation, lethargy, poor peripheral perfusion, respiratory distress, blood sugar fluctuations and metabolic acidosis, whether or not accompanied by bacteraemia, in the initial 4 weeks of life ${ }^{1}$. It may be separated into early onset sepsis (presenting in first 72 hours of life) and late onset sepsis (presenting after first 72 hours of life) $)^{2}$. Knowledge, experience and vigilance are needed for identification of subtle signs early to prevent neonatal disability or death. Whilst antibiotic therapy is curative, early diagnosis and management are important to prevent untoward effects $^{3}$. Nurses use many ways to assess and identify signs and symptoms of sepsis. Approaches used by a nurse in the identification of the sick state of an infant may be diverse $^{3}$. Nurses' knowledge and input are critical for early recognition of sepsis in all settings ${ }^{3}$.

Sepsis is still one of the chief causes of mortality and morbidity, especially in the initial five days of life and in low and middle-income countries ${ }^{4}$. In 2012, around 7 million severe bacterial infections occurred in neonates in South Asia, sub-Saharan Africa, and Latin America ${ }^{4}$. In 2015, of 5.941 million deaths in the under the 5 year olds, $45 \%$ died in the newborn period and sepsis was the third commonest cause of death with around 0.4 million deaths, most of which were in developing countries $^{5}$. There has been little research carried out on this topic and so far none in Sri Lanka. Thus evaluation of nurses' knowledge of neonatal sepsis 
is timely and of benefit in improving quality of care provided to the newborn.

\section{Objectives}

The general study objective was to assess the nurses' knowledge regarding neonatal sepsis. The specific objectives were to find out the association between knowledge regarding sepsis and other factors, namely, place of work, duration of exposure to newborn care and institute of training.

\section{Method}

Design: Descriptive cross-sectional study

Setting and population: All nursing officers of the neonatal intensive care unit (NICU), post-natal wards, paediatric ward and the labour ward were included in the study excluding the trainee nurses. They were invited with the permission of the Director and the Matron of the hospital.

Duration of study: The study was carried out over a period of 2 months.

Study instrument: An interviewer-administered questionnaire was utilised for data collection. All forms were completed during individual interview by the investigators. Questionnaire included six components to cover the understanding of neonatal sepsis, causes for neonatal sepsis, risk factors, clinical features, actions upon identification of sepsis and consequences of missing sepsis. Overall knowledge was calculated using the scores obtained in each section of the questionnaire.

Analysis: Data were analysed using SPSS 20. Results were graded according to the cumulative score.

Good knowledge $\quad=>8$

Satisfactory knowledge $=5-8$

Unsatisfactory knowledge $=<5$

Ethical issues: The aim of the study was explained to each nurse individually and written consent obtained. All nurses willingly participated with the first explanation. Ethical clearance was obtained from the ethics review committee of Sri Jayewardenepura General Hospital.

\section{Results}

A total of 81 nursing officers participated in the study. This included all nurses except those on maternity leave. They were categorized according to the unit of work, educational institute and the duration of exposure to neonatal care (Table 1).

Overall knowledge was calculated using the scores obtained in each section of the questionnaire. (Table 2)

Table 1: Categorisation of nurses

\begin{tabular}{|l|c|c|c|c|c|c|c|}
\hline \multirow{2}{*}{ Unit } & \multirow{2}{*}{ No. } & \multicolumn{2}{|c|}{ Educational institute } & \multicolumn{4}{c|}{ Duration of exposure } \\
\cline { 3 - 8 } & & NTS & $\begin{array}{c}\text { University } \\
\text { (BSc) }\end{array}$ & $<\mathbf{6}$ months & $\begin{array}{c}\text { 6 months } \\
\text { to 1 year }\end{array}$ & $\begin{array}{c}\text { >1 year to 3 } \\
\text { years }\end{array}$ & $>3$ years \\
\hline NICU & 21 & 20 & 01 & 0 & 03 & 02 & 16 \\
\hline Post-natal wards & 36 & 29 & 07 & 05 & 03 & 06 & 22 \\
\hline Labour room & 14 & 14 & 0 & 0 & 0 & 01 & 13 \\
\hline Paediatric ward & 10 & 10 & 0 & 0 & 0 & 05 & 05 \\
\hline
\end{tabular}

Table 2: Overall knowledge on different aspects of neonatal sepsis

\begin{tabular}{|l|l|c|}
\hline \multicolumn{1}{|c|}{ Question } & \multicolumn{1}{|c|}{ Knowledge } & Frequency (\%) \\
\hline Meaning of neonatal sepsis & Good (2) & $73(90.1)$ \\
& Satisfactory (1) & $04(04.9)$ \\
& Unsatisfactory (0) & $04(04.9)$ \\
\hline Causes of neonatal sepsis & Good (>2) & $76(93.8)$ \\
& Satisfactory (2) & $05(06.2)$ \\
& Unsatisfactory $(<2)$ & $0(0)$ \\
\hline Risk factors of neonatal sepsis & Good (>3) & $06(07.4)$ \\
& Satisfactory $(2-3)$ & $59(72.8)$ \\
& Unsatisfactory $(<2)$ & $16(19.8)$ \\
\hline Clinical features of neonatal sepsis & Good (>3) & $58(71.6)$ \\
& Satisfactory $(2-3)$ & $22(27.1)$ \\
& Unsatisfactory $(<2)$ & $01(01.2)$ \\
\hline Actions upon identification of sepsis & Good (1) & $69(85.2)$ \\
& Unsatisfactory $(0)$ & $12(14.8)$ \\
\hline Consequences of missing sepsis & Good $(1)$ & $79(97.5)$ \\
& Unsatisfactory $(0)$ & $02(02.5)$ \\
\hline
\end{tabular}


Ninety percent and $4.9 \%$ of nurses had good and satisfactory knowledge respectively as to what neonatal sepsis means, whereas $4.9 \%$ had no acceptable knowledge. Out of the nurses whose knowledge was good or satisfactory, $76.6 \%$ knew about septicaemia but only $28.5 \%$ knew about meningitis. All participants (100\%) knew bacteria as causative organisms of neonatal sepsis, but only $81.5 \%$ knew about viruses and $64.2 \%$ came up with fungi as causative organisms (Table 3). Most participants from NICU (19/21) knew fungi as a causative organism in contrast to few from labour room (5/14) and the paediatric unit (5/10). 6/8 BSc nurses and 46/73 NTS nurses knew fungi as a causative organism. All the nurses with exposure to newborn care $<1$ year knew fungi as a causative organism whereas $10 / 14(71.4 \%)$ and $24 / 56$ $(42.8 \%)$ knew it in $1-3$ year exposure and $>3$ years exposure ranges respectively.

Table 3: Organisms causing neonatal sepsis

\begin{tabular}{|l|c|}
\hline \multicolumn{1}{|c|}{ Organism } & Frequency (\%) \\
\hline Bacteria & $81(100)$ \\
\hline Virus & $66(81.5)$ \\
\hline Fungi & $52(64.2)$ \\
\hline
\end{tabular}

Regarding the knowledge on risk factors of neonatal sepsis, majority $(80.2 \%)$ had a satisfactory or good knowledge and $19.8 \%$ had an unsatisfactory knowledge. $17 / 21$ participants in NICU, 13/14 in labour room, 32/36 in postnatal units and $3 / 10$ in paediatric ward had good or satisfactory knowledge. The knowledge of $70 \%$ of nurses in the paediatric ward was unsatisfactory. Out of the participants who had an unsatisfactory knowledge only one nurse had less than one-year exposure to neonatal care. All BSc nurses had good or satisfactory knowledge about risk factors for neonatal sepsis.

Risk factors for neonatal sepsis are shown in Table 4. Of the nurses $76.5 \%$ identified pre-labour rupture of membranes as a risk factor and $60.5 \%$ identified intrapartum maternal pyrexia. Only $23.5 \%$ knew prematurity as a risk factor. Even though intrapartum minimum handling is very important in preventing early onset neonatal sepsis only $16 \%$ identified that as a risk factor. More importantly only $35.8 \%$ came up with poor hand hygiene as a risk factor for neonatal sepsis.

Table 4: Risk factors for neonatal sepsis

\begin{tabular}{|l|c|}
\hline \multicolumn{1}{|c|}{ Risk factor } & Frequency (\%) \\
\hline $\begin{array}{l}\text { Premature rupture of } \\
\text { membranes }\end{array}$ & $62(76.5)$ \\
\hline $\begin{array}{l}\text { Intrapartum maternal } \\
\text { pyrexia and infections }\end{array}$ & $49(60.5)$ \\
\hline Prematurity & $19(23.5)$ \\
\hline Intrapartum handling & $13(16.0)$ \\
\hline Poor hand hygiene & $29(35.8)$ \\
\hline
\end{tabular}

Regarding the identification of signs and symptoms of neonatal sepsis, the majority (98.7\%) had a good or satisfactory knowledge on signs and symptoms of neonatal sepsis whereas only one participant had an unsatisfactory knowledge.

Identification of symptoms and signs of neonatal sepsis are shown in Table 5. Majority $(92.6 \%)$ knew fever as a sign of neonatal sepsis but only $19.8 \%$ knew of hypothermia. Out of the 16 nurses who knew hypothermia as a sign of neonatal sepsis $9(56.3 \%)$ were working in the NICU while $2.7 \%$ were from postnatal units. All the nurses who came up with hypothermia as a sign were trained in nursing training school. $85.8 \%$ knew feeding problems as a symptom of neonatal sepsis and $72.8 \%$ came up with lethargy. 39.5\% knew about respiratory distress in early onset neonatal sepsis. Only $21 \%$ knew about RBS fluctuations in neonatal sepsis.

Table 5

Identification of symptoms and signs of neonatal sepsis

\begin{tabular}{|l|c|}
\hline Symptom/Sign & Frequency (\%) \\
\hline Fever & $75(92.6)$ \\
\hline Feeding problems & $69(85.2)$ \\
\hline Lethargy & $59(72.8)$ \\
\hline Skin mottling & $42(51.9)$ \\
\hline Baby not looking alright & $40(49.4)$ \\
\hline Respiratory distress & $32(39.5)$ \\
\hline RBS fluctuations & $17(21.0)$ \\
\hline Hypothermia & $16(19.8)$ \\
\hline
\end{tabular}

$85.5 \%$ of the nurses knew what action had to be taken upon identification of neonatal sepsis and the rest $(14.2 \%)$ had no idea. All the nurses with less than 1 year exposure and all the BSc nurses knew the actions to be taken on identification of neonatal sepsis. Seventy nine (97.5\%) participants had a good knowledge on consequences of missing a neonatal sepsis. Rest, who did not know the consequences were from the postnatal units.

According to the study $72.8 \%$ of nurses had an overall good knowledge on neonatal sepsis and the rest $(27.2 \%)$ had a satisfactory knowledge (Table 6). No one had an unsatisfactory knowledge. $76.1 \%$ of NICU nurses, $80.5 \%$ of postnatal unit nurses, $78.5 \%$ of labour ward nurses had a good knowledge on neonatal sepsis whereas only $30 \%$ of the nurses working in paediatric unit had a good knowledge. All the nurses with a working experience less than 1 year had a good knowledge while $57.1 \%$ of nurses with working experience of 1-3 years and $71.4 \%$ of nurses with working experience more than 3 years had a good knowledge. $87.5 \%$ of BSc degree holding nurses had a good knowledge on neonatal sepsis, whereas 
$71.2 \%$ of nurses with NTS training had a good knowledge.

Table 6: Overall knowledge on neonatal sepsis

\begin{tabular}{|l|c|}
\hline \multicolumn{1}{|c|}{ Overall knowledge } & Frequency (\%) \\
\hline Good knowledge $(>8)$ & $59(72.8)$ \\
\hline Satisfactory knowledge (5-8) & $22(27.2)$ \\
\hline Unsatisfactory knowledge $(<5)$ & $0(0)$ \\
\hline
\end{tabular}

\section{Discussion}

This study revealed that all nurses involved in neonatal care had good or satisfactory overall knowledge on neonatal sepsis. This is a good indicator in comparison to a similar study conducted in Baghdad, where, only $62.7 \%$ of nurses had acceptable knowledge ${ }^{6}$. However, knowledge deficiencies were identified regarding risk factors for neonatal sepsis, the cues in identifying sepsis and initial action upon identification of sepsis. While all nurses had satisfactory knowledge regarding the causative organisms, wider knowledge was noted among NICU and BSc qualified groups indicating knowledge gained via case exposure and probable improved curricula. Interestingly, knowledge regarding aetiology was inversely proportional to the duration of neonatal care experience, reflecting lack of education programmes and updated lectures for nurses.

Regarding the risk factors for neonatal sepsis, a significant proportion (19.8\%) had an unsatisfactory knowledge. Only $35.8 \%$ voiced poor hand hygiene as a risk factor for newborn sepsis. This is an important finding in the study, prompting the need for more awareness programmes regarding hand hygiene as it is considered the most important step in prevention of neonatal sepsis ${ }^{7}$. Boettiger et al considered nurses' ability in identifying physiologic and behavioural indicators associated newborn sepsis as a valuable clinical tool $^{8}$. He also concluded that recognising these indicators was related more to working in the NICU than the level of nursing education ${ }^{8}$. Our study showed good or satisfactory knowledge in almost all nurses across the units of work. However, in our study only a few knew about hypothermia which is an important sign of sepsis. Majority identified feeding problems, lethargy and fever as symptoms but only a minority came up with respiratory distress, hypothermia, blood sugar fluctuations and skin mottling.

Majority knew that the doctor should be informed / antibiotics should be started once sepsis is suspected, but $14.8 \%$ of the participants gave unsatisfactory responses indicating gaps in knowledge. Most of the participants (97.5\%) were well aware of the consequences of missing neonatal sepsis with only 2 participants responding poorly. This knowledge will help in getting them trained to share the responsibility of identification and management. Even though the overall knowledge turned out to be good or satisfactory in all the participants there were areas to which more attention should be paid. No significant association was found between the knowledge on neonatal sepsis and the institute of training or duration of exposure $(p>0.05)$. However, some degree of association was tracked between the unit of work and knowledge on sepsis.

This study covered most of the important aspects of knowledge on neonatal sepsis, yet further research on this topic would be of value in identifying further deficiencies in knowledge and to bridge the gaps. Since nursing officers are the frontline assessors of the newborns, their knowledge on neonatal sepsis and early recognition play a key role in management of this life-threatening condition. A study conducted by Yousefi et al in Iran found improvement in attitude, knowledge, and practice of sepsis care among ICU nurses following an educational programme ${ }^{9}$. Thus, regular training programmes would be beneficial in raising awareness on neonatal sepsis with the aim of reducing the global burden of its morbidity and mortality.

\section{Conclusions}

Seventy three percent of the nurses had an overall good knowledge on neonatal sepsis and the rest had a satisfactory knowledge. Knowledge deficiencies were identified regarding risk factors for neonatal sepsis, initial action upon identification of sepsis and the cues in identifying sepsis. No significant association was found between the knowledge on neonatal sepsis and the institute of training or duration of exposure. However, some degree of association was tracked between the unit of work and knowledge on sepsis.

\section{References}

1. Sepsis in the newborn. All India Institute of Medical Sciences (AIIMS) protocol. 2014. CBS Publishers, Delhi.

2. Singh M, Narang A, Bhakoo ON. Predictive perinatal score in the diagnosis of neonatal sepsis. Journal of Tropical Pediatrics 1994; 40(6):365-8. https://doi.org/10.1093/tropej/40.6.365 PMid: 7853443

3. Rubarth L. Nursing patterns of knowing in assessment of newborn sepsis. University of Arizona; 2005. 
4. Seale A.C, Blencowe H, Manu A A, Nair $\mathrm{H}$, Bahl R, Qazi S A et al. Estimates of possible severe bacterial infection in neonates in sub-Saharan Africa, south Asia, and Latin America for 2012: a systematic review and meta-analysis. Lancet Infectious Diseases 2014; 14(8):731-41.

https://doi.org/10.1016/S14733099(14)70 804-7

5. Liu L, Oza S, Hogan D, Yue C, Perin J, Zhu J et al. Global, regional, and national causes of under-5 mortality in 2000-15: an updated systematic analysis with implications for the Sustainable Development Goals. Lancet 2016. https://doi.org/10.1016/S01406736(16)31 593-8

6. Obaid KB, Hussein A. Nurses' knowledge concerning neonatal sepsis in neonatal intensive care units at paediatric teaching hospitals in Baghdad city. Asian Academic Research Journal of Multidisciplinary 2016; 3(7):56-65.
7. Sharma G, Zaka N, Hailegebriel T. Infection Prevention Control at Neonatal Intensive Care Units. UNICEF: New York. 2018. Available from: https://www.healthynewbornnetwork.org/ hnn-content/uploads/Infection-Preventionand-Control-at-NICU-Slide-Deck2.8.2018.pdf

8. Boettiger M, Tyer-Viola L, Hagan J. Nurses' early recognition of neonatal sepsis. Journal of Obstetric, Gynecologic, and Neonatal Nursing 2017; 46(6):83445.

https://doi.org/10.1016/j.jogn.2017.08.007 PMid: 28987479

9. Yousefi, H., Nahidian, M, Sabouhi R. Reviewing the effects of an educational program about sepsis care on knowledge, attitude, and practice of nurses in intensive care units. Iranian Journal of Nursing and Midwifery Research 2012; 17(2 Suppl 1); S91-5.

PMid: 23833608 PMCid: PMC3696963 